\title{
Evolution of Localization in Pseudoelastic NiTi Tubes Under Biaxial Stress States
}

by

\author{
Nathan J. Bechle and Stelios Kyriakides ${ }^{1}$
}

1 Corresponding author

e-mail: $\underline{\text { skk@mail.utexas.edu }}$

Tel. 512-471-4167

Key Words:

Shape Memory Alloys, Pseudoelasticity, Biaxial Loadings, Localization, Inhomogeneous Deformation

International Journal of Plasticity

Accepted January 2016

Research Center for Mechanics of Solids, Structures \& Materials WRW 110, The University of Texas at Austin

Austin, Texas 78712 


\section{ABSTRACT}

Nearly equiatomic NiTi can be strained in tension to several percent and fully recover upon unloading (pseudoelastic behavior). This property is derived from solid-state transformations between the austenitic (A) and martensitic (M) phases, which can be induced by either changes in temperature or stress. It is well established that stress-induced phase transformation leads to localized deformation associated with the nucleation and propagation of the M-phase during loading and the A-phase during unloading. It has been demonstrated that this partially unstable material behavior strongly influences the stability of NiTi structures, motivating the need for the development of constitutive models that can capture this instability. To support the calibration of such constitutive models, the behavior of $6.35 \mathrm{~mm}$ diameter pseudoelastic NiTi tubes loaded under combined axial load and internal pressure has been examined. Tubes were loaded under radial stress paths in the axial-hoop stress space using stereo digital image correlation for fullfield monitoring of the evolution of transformation-induced deformation. Results from 26 experiments spanning axial-to-hoop stress ratios from -1.0 to uniaxial tension revealed that, but for a narrow region near equibiaxial tension, transformation leads to localized helical deformation bands with inclinations dependent on the stress ratio. In the process, the stresses remain nearly constant until transformation is completed. In the vicinity of equibiaxial tension, the material exhibits hardening and homogeneous deformation. Loci of the transformation stresses, while exhibiting very modest anisotropy, traced an elongated trajectory in the axialhoop stress space not captured by a von Mises representation. By contrast, the transformation strains exhibited significant anisotropy between axial and hoop dominant stress paths. Moreover, strains around the equibiaxial stress state, where material hardening and homogeneous deformation was observed, were significantly smaller than in the rest of the stress space. The strain anisotropy had a corresponding reflection on the energy dissipated during transformation with axial dominant stress paths dissipating significantly more energy than hoop dominant ones, with less dissipation observed in the neighborhood of equibiaxial stress. 


\section{INTRODUCTION}

One of the unique properties of shape memory alloys (SMAs) is pseudoelasticity, which describes the ability of the material to be deformed to strains of a few percent that are fully recovered on unloading ( $\sim 7 \%$ for nearly equiatomic NiTi). This property is derived from a diffusionless transformation between the austenitic (A) and martensitic (M) phases, which can be induced by either changes in temperature or stress. It is well established that under tension, stress-induced phase transformation leads to localized deformation where "highly" strained Mphase coexists with relatively low-strained A-phase at the same stress (e.g., Shaw and Kyriakides [1995, 1997], Liu et al. [1999], Li and Sun [2002], Sun and Li [2002], Iadicola and Shaw [2002, 2004], Feng and Sun [2006], Abeyaratne and Knowles [2006], Favier et al. [2007], Daly et al. [2007], Hallai and Kyriakides [2013], Reedlunn et al. [2014], Bechle and Kyriakides [2014]).

Under compression the behavior of SMAs, although less studied because of experimental challenges, can be summarized as follows: the material again exhibits pseudoelasticity but the transformation stress is higher, the strain is smaller, the response is monotonic, and the deformation is essentially homogeneous. (e.g., Wasilewski [1971], Patoor et al. [1995], Jacobus et al. [1996], Orgeas and Favier [1998], Gall and Sehitoglu [1999], Gall et al. [1999], Sehitoglu et al. [2000], Thamburaja and Anand [2002], Mao et al. [2010], Reedlunn et al. [2014], Bechle and Kyriakides [2014]). The consensus is that the main cause of this difference is due to crystallographic texture.

Although pseudoelasticity and the other unique property of SMAs, the shape memory effect, have been exploited in practical applications such as biomedical devices, aerospace structures, seismic isolation/damping devices, MEMS, and others, it is fair to say that at the present the use of SMAs remains limited. Research efforts that combine these unique characteristics with structural nonlinearities to produce novel applications include the following: Michailidis et al. [2009] demonstrated the kinematic amplification and the reversibility of crushing afforded by the use of a SMA in a honeycomb crushed in-plane. Rahman et al. [2001] showed that SMA columns buckle at the expected load, but under continued compression can recover stiffness. Jiang et al. [2015] examined the energy absorption potential of pseudoelastic NiTi shells that under axial compression buckle, collapse, and recover (see also Nemat-Nasser et al. [2006], Tang and Li [2012]). Bechle and Kyriakides [2014] demonstrated that NiTi tubes 
under bending develop localization and propagation of curvature that is recoverable upon unloading (see also Reedlunn et al., 2014).

A dire requirement for the wider use of SMAs in these and other applications is constitutive models that capture their complex behavior. The main challenges include: (a) the inhomogeneous deformation associated with phase transformation in the pseudoelastic temperature regime, hitherto observed primarily under uniaxial tension; (b) the significant tension compression asymmetry exhibited by processed NiTi structural bulk materials; and (c) the thermomechanical interactions that take place. A number of constitutive models developed during the last decade are capable of capturing some of these complexities but not all (e.g., Auricchio and Petrini [2004], Govindjee and Hall [2000], Govindjee and Miehe [2001], Popov and Lagoudas [2007], Hartl and Lagoudas [2009], Arghavani et al. [2010], among others). Although success in reproducing some SMA behavior is demonstrated by many of these constitutive models their wider applicability is limited or unproven. A primary reason is the present lack of dependable data about the behavior of SMAs under multiaxial loadings that can be used to calibrate and test proposed constitutive models.

Efforts to establish the response of NiTi under biaxial stress states are limited and those performed have mainly concentrated on establishing the onset of transformation under tension and shear usually using tubes under combined tension and torsion (e.g., Lim and McDowell [1999], Helm and Haupt [2001], Sun and Li [2002], Bouvet et al. [2004], Lexcellent and Blanc [2004], McNaney et al. [2003], Grabe and Bruhns [2009], Shaw et al. [2013]). Other contributions to the subject are those of Orgeas and Favier [1998], Thamburaja and Anand [2002], and a body of work from Lexcellent and co-workers using mostly $\mathrm{Cu}$ based SMAs (e.g., Rogueda et al. [1996], Lexcellent et al. [2002], Bouvet et al. [2002]). The main conclusions from this body of work are summarized in Sun and Li [2002] who conducted series of experiments on NiTi tubes involving tension under different values of constant torque, and torsion experiments under different values of tension: (i) NiTi under shear exhibits a monotonically increasing response with limited or no localization. (ii) Tension dominant stress states lead to localization and shear dominant ones do not. (iii) The nucleation of $\mathrm{M}$ transformation surface does not follow a von Mises type trajectory.

This study aims to address the need for a more complete picture of how pseudoelastic NiTi behaves under biaxial stress states. To this end a custom internal pressure-axial load testing 
facility is used to conduct a series of experiments on NiTi tubes under radial paths in the axialhoop stress space. For each stress path the specimen is loaded under nearly isothermal conditions to the "completion" of martensitic transformation and unloaded. Stereo Digital Image Correlation (DIC) is used to monitor and capture the real-time deformation of the specimen. The issues of interest include: (i) How does biaxiality influence the onset of $M$ transformation during loading and the onset of A transformation during unloading. (ii) Identify any prevalent anisotropies in stress or strain. (iii) How does the material deform during transformation under different stress states. (iv) How is the hysteresis or energy dissipated influenced by biaxiality.

\section{EXPERIMENTAL}

The experiments are conducted on tubular specimens from a nearly equiatomic NiTi tubular stock $(50.9$ at $\% \mathrm{Ni}$ ) supplied by Memry Corporation. The tubes are produced by drawing followed by thermomechanical processing that enhances the special properties of this alloy. The tube stock is then finished by centerless grinding to a specified outer diameter. This is the same tubular stock used in the axial buckling study of Jiang et al. [2015]. The tubes had a specified outer diameter of $6.35 \mathrm{~mm}(0.25 \mathrm{in})$ and a wall thickness of approximately $0.25 \mathrm{~mm}$ $(0.010 \mathrm{in})$-the exact dimensions of the specimens are listed in Table 1. The following transformation temperatures were established for the material using digital scanning calorimetry: $A_{s}=-10{ }^{\circ} \mathrm{C}$ and $A_{f}=12{ }^{\circ} \mathrm{C}$. The experiments were performed at room temperature $\left(23^{\circ} \mathrm{C}\right)$ at which the material is in the austenitic phase and exhibits pseudoelasticity.

The biaxial testing facility consists of a screw-type axial testing machine that operates in conjunction with a 690 bar (10,000 psi) precision metering syringe pump using water as the pressurizing fluid. The pump has an independent controller that allows either pressure or volume control. A unique feature, essential to the present application, is that the syringe pump can dispense very small volumes at precision flow rates. The specimen is mounted into the testing machine via custom stainless-steel housing grips as shown in Fig. 1. The upper housing is connected to a $5 \mathrm{kN}(1000 \mathrm{lb})$ load cell while the lower one is anchored to the crosshead of the testing machine. Careful alignment of the two housings is performed prior to mounting the specimen.

The ends of the specimen are clamped in the housings using self-centering 1-inch locking assemblies as shown in Fig. 2. Solid spacers that allow the required contraction on clamping are 
used to fill the space between the locking assemblies and the specimen. The specimen is protected from crushing by steel plugs inserted into the clamped ends to react the clamping force. Centered holes drilled along the length of the plug allow free flow of the pressurizing fluid. Double O-rings are used to seal the plug against the specimen and against the housing as shown schematically in Fig. 2.

One-eighth-inch high-pressure tubing and connectors are used to connect the pump to the lower housing. The hydraulic circuit is completed with valves at the entrance and the exit (exhaust) as shown schematically in Fig. 1a (see also photograph in Fig. 1b). The pressure in the system is monitored with a pressure transducer calibrated to $10 \mathrm{~V}$ at 10000 psi.

The specimens were cut from a $1525 \mathrm{~mm}$ (5 ft) long tube stock to a length of $114 \mathrm{~mm}$ (4.50 in). The ends were faced off in a lathe and deburred to ensure that the O-rings are not damaged during the insertion of the end plugs. Clamping leaves a $50 \mathrm{~mm}(2 \mathrm{in})$ test section. A fine black speckle pattern is painted on a white undercoat on the test section to enable monitoring the deformation during the test using stereo Digital Image Correlation (DIC).

We report experiments in which the specimens were loaded along radial stress paths such that the nominal axial $(x)$ and hoop $(\theta)$ stresses are related via

$$
\sigma_{X}=\alpha \sigma_{\theta}, \quad \alpha=\text { const. }
$$

This is achieved by connecting the axial and pressure loading systems through feedback as follows. Let $F$ and $P$ be respectively the force measured by the load cell and the internal pressure. The axial and circumferential stresses are then

$$
\sigma_{X}=\frac{F}{2 \pi R t}+\frac{P R}{2 t} \text { and } \sigma_{\theta}=\frac{P R}{t},
$$

where $R$ and $t$ are respectively the undeformed mid-surface radius and wall thickness of the tube. Thus, for example, if the load cell reads zero force it means that the specimen is reacting the pressure end-cap load and consequently develops an axial stress of $P R / 2 t(\Rightarrow \alpha=0.5)$. Pressurization was performed by prescribing the fluid flux into the closed system (volume control). The axial testing machine is operated under load control with the pressure signal as external command using a TestProfiler method in Instron's Bluehill 3 software. The pressure is used in Eqs. (1) and (2) to establish a target value of force that corresponds to the chosen value 
of $\alpha$. The target value of force is compared to the current set point and the difference becomes the command signal that ramps the force in a chosen time step (typically $1 \mathrm{~s}$ ). In this manner the force is continuously updated so that it follows the measured pressure at the desired ratio. Experiments were performed for $-1.0 \leq \alpha \leq \infty$.

During the test the pressure, displaced volume, axial load, and machine displacement are monitored using a LabVIEW based data acquisition system. Each channel is sampled at $10 \mathrm{kHz}$ and the signals are averaged over 1000 samples and stored at a rate of 10 per second.

Because inhomogeneous deformations were anticipated to develop for parts of the loading histories, a full-field deformation monitoring scheme was adopted. Stereo Digital Image Correlation (DIC) was used to record the surface deformation history over the full $50 \mathrm{~mm}$ length of the test section and approximately one third of its circumference. An ARAMIS based DIC system with two $5 \mathrm{MP}$ cameras was used. A facet size of $24 \times 24$ pixels $(\sim 0.28 \times 0.28 \mathrm{~mm})$ and spacing of 12 pixels $(\sim 0.14 \square \mathrm{m})$ were used providing the field of view (FOV). Typically a stage was recorded every 5 seconds. Post-processing of the recorded stages allows determination of the axial and circumferential engineering strains $\varepsilon_{X}$ and $\varepsilon_{\theta}$.

As is well established, latent heat associated with transformation-induced deformation can heat/cool the specimen, changing the recorded stress. It is thus imperative that in material characterization experiments stresses be recorded under isothermal conditions (Shaw and Kyriakides, 1995; Bechle and Kyriakides, 2014). This is usually accomplished by performing the tests in a circulating liquid bath at a slow strain rate or, in the case of compression, by passing a liquid through the specimen at a constant flow rate. Because of constraints placed by the optical diagnostic techniques used in the present experiments, surrounding the specimen with water was not possible and so instead the strain rate was maintained at a slow enough level to ensure that any released heat was dissipated by conduction and radiation. In the biaxial experiments performed, the rate of loading was determined by the rate at which water was pumped into the system, which was typically at $0.02 \mathrm{ml} / \mathrm{min}$.

The adequacy of this arrangement was checked by monitoring the temperature of the deforming specimen with a thermocouple. For select experiments the temperature of the full test section was monitored with an infrared camera. For most of the experiments, the $0.02 \mathrm{ml} / \mathrm{min}$ flow rate proved to be adequate in that temperature changes were kept below $\pm 1^{\circ} \mathrm{C}$. However, for higher values of the stress ratio $\alpha$, the required volume change is relatively small. In such 
cases, volume-control pressurization is not ideal causing the induced, mainly axial, localized deformation to develop rather rapidly. This in turn results in modest increase/decrease in the local temperature of up to $\pm 3{ }^{\circ} \mathrm{C}$. Such temperature changes will be reported in the results that follow.

\section{RESULTS FROM TWO REPRESENTATIVE EXPERIMENTS}

Results from two representative experiments are now used to describe the material response to radial $\sigma_{X}-\sigma_{\theta}$ loading histories. In order to connect to the uniaxial experiments reported in Bechle and Kyriakides [2014], we start with the uniaxial tension experiment. Results from a radial biaxial experiment with $\sigma_{X}=0.375 \sigma_{\theta}$ will follow.

\section{a. Uniaxial Tension Test: $\sigma_{\theta}=0$}

In this case the specimen was pulled under displacement control at a rate that induced an axial strain rate of $5 \times 10^{-5} \mathrm{~s}^{-1}$ when deformation was homogeneous. Figure 3 shows the nominal stress-mean strain $(\sigma-\bar{\varepsilon})$ responses recorded in the axial, $x$, and hoop, $\theta$, directions. The "mean" strain in each direction represents the spatial average over the full of $320 \times 60$ facet FOV monitored (measurement accuracy $\left.\bar{\varepsilon}_{X} \sim|0.04 \%|, \quad \bar{\varepsilon}_{\theta} \sim|0.09 \%|\right)$ ). Figure 4 shows 20 sets of fullfield DIC contours of axial and hoop strain corresponding to the numbered bullets on the responses in Fig. 3.

The response exhibits an initial stiff and nearly linear branch during which the $A$ phase deforms elastically and homogeneously as depicted in images (1). From this part of the response the elastic modulus, $E_{A}$, is measured to be $65.7 \mathrm{GPa}(9.53 \mathrm{Msi})$ and the Poison's ratio, $v_{A}=0.424$ (see additional results in Appendix C). A small reduction in the modulus is observed at stress values higher than $400 \mathrm{MPa}$ indicating that some small amount of material is starting to transform (see Shaw and Kyriakides, 1995). However, the deformation remains homogeneous up to a stress of $458 \mathrm{MPa}$ when two helical higher deformation band-like patterns initiate from the two gripped ends (2) (compares with transformation stress of $381 \mathrm{MPa}$ of the tension test of Bechle and Kyriakides, 2014). The two helixes follow opposite directions, presumably along the two Hill [1952] characteristics, but the bands make the same angle with the axial direction. They grow by forming helical patterns around the tube surface (3) with their strain quickly growing to 
levels close to $7 \%$ that is associated with martensitic transformation. The tips of the propagating helical bands are relatively pointed while behind the bands tend to broaden. As expected, the gradual propagation of $M$ takes place with the stress remaining nearly constant.

A detailed evolution of the patterned localized transformation is best visualized from the complete record in the format of a video, which can be found on $\mathrm{x}$-Tension under Supplementary Data. The major events can be summarized as follows:

- By image (4), propagation of the tip of the top band is halted and a set of additional helical patterns initiate from the transformed region behind the leading band with approximately the same orientation as the parent front. The multi-helical front propagates as shown in images (5) -8. Such multi-helical patterns are often preferred as they tend to minimize structural distortion to the tubular specimen.

- In the neighborhood of image $9\left(\bar{\varepsilon}_{X} \sim 6.5 \%\right)$, the top and bottom fronts are about to coalesce and this leads to the tracing of the customary stress valley.

- By point (10, the stress has taken an upswing and the corresponding images show the deformation to be again uniform at a level of just over 7\%.

Unloading commences at this point at the same displacement rate. Initially, deformation is once more uniform (11) and (12). At a stress of $234 \mathrm{MPa}$ (33.9 ksi) $\mathrm{M} \rightarrow \mathrm{A}$ transformation initiates at the lower end in the form of a multi-helical front with a single pointed band connecting it to the bottom end (see image ${ }^{13}$; see detailed study of such fronts in Feng and Sun [2006]). In images (13) and (14) the front propagates leaving behind material that has transformed to A at a strain corresponding to the end of the lower plateau. At $\bar{\varepsilon}_{X} \sim 4.5 \%$, close to image (14), an additional front appears in the FOV at the top of the specimen developing again a multi-helical front with the opposite chirality-handedness (see (15). The two fronts propagate simultaneously eventually coalescing, which leads to the usual small stress peak. Subsequently the specimen, now fully transformed to the A-phase, unloads with the A elastic modulus returning to zero stress at a strain of $0.08 \%$ (this residual strain is of the order of the accuracy of the DIC measurements.)

A more quantitative measure of the extent of inhomogeneity in the specimen is established as follows. A number of equally spaced "bins" of strain levels spanning the range of strains recorded are first established. For each DIC data set the bins are populated with the number of points with the corresponding strain range from the entire $320 \times 60$ facet field. The resultant frequencies are then smoothed using a Gaussian filter forming a continuous 
distribution, and the smoothened frequency distributions are normalized such that the peak frequency is unity. Figure 5 shows such histograms of axial strain corresponding to the 20 images in Fig. 4. The histogram for image (1) in which the deformation is homogeneous exhibits a relatively narrow unimodal distribution corresponding to the current strain level. For image (3), a bimodal distribution begins to develop with the second mode centered around a strain of about $7 \%$, due to coexistence of two deformation regimes. As $M$ propagates through the specimen, the amplitude of the higher strain mode grows while that of the lower strain gradually reduces. On the completion of the transformation (10) the small strain peak disappears and the distribution becomes unimodal again. The peak centered around 7\% strain reaches its highest amplitude and its narrowest form. Such histograms have been generated for both axial and hoop strains for each experiment. (Note that the histograms are based only on the DIC FOV.)

An alternative method of relating the temporal and spatial evolution of transformation is as follows. The axial position of the front(s) is established from the image record. Used together with the time of the image an $x$ - $t$ diagram of the evolution of the front is constructed. In tension experiments on small diameter SMA rods and strips (Shaw and Kyriakides 1995; 1997) and steel strips that develop Lüders bands (Butler, 1962; Kyriakides and Miller, 2000), such diagrams revealed that fronts propagated continuously until the whole specimen was transformed. The velocity of the front, $c$, could then be related to the applied displacement rate, $\dot{\delta}$, and the transformation (or Lüders) strain, $\Delta \varepsilon^{t}$, as follows:

$$
c=\frac{\dot{\delta}}{n \Delta \varepsilon^{t}}
$$

where $n$ is the number of active propagating fronts. The evolution of transformation in the tubular specimen is complicated by transitions from single to multi-helical fronts, temporary halts of propagation at specific sites, transformation outside the FOV, etc. Such complications made tracking of the evolution of fronts during the $\mathrm{A} \rightarrow \mathrm{M}$ stress plateau difficult. However, the $\mathrm{M} \rightarrow \mathrm{A}$ transformation progressed in a more orderly manner, which enabled tracking of the position of the center of the front in the FOV and construction of the $x$-t diagram shown in Fig. 6. Included is the average strain corresponding to the time axis (note that $\bar{\varepsilon}_{X}$ is not directly proportional to $t$ ). The diagram is broken up into several data sets corresponding to distinct events observed in the FOV. Data sets 2 and 3 correspond to the upward propagation of the 
multi-helical front at the lower end of the specimen. The two data sets trace linear trajectories with slopes, or speed of the propagation, much lower than the value of a single front propagation yielded by (3) - with $\Delta \varepsilon^{t}=0.054$. This is an indication that additional transformation activity is taking place outside the FOV (with data set 2 also seeing the effect of simultaneous single and multi-helical fronts).

Data set 4 corresponds to the appearance and propagation of the upper front in the FOV. Its velocity is lower than the corresponding part of set 3, but interestingly the average of the propagation velocities of 3 and 4 is close to the value yielded by (3) for $n=2$. Data sets 5 and 6 correspond to the propagation of the upper and lower multi-helical fronts toward each other (e.g., images 16 and 17 in Fig. 4). The speed of the lower one is significantly higher than that of the upper one, but the average velocity again approximately coincides with that yielded by (3) for $n$ $=2$. Thus unlike observations of fronts made on strips and small diameter rods where fronts tended to propagate simultaneously at essentially the same speed, in the present tubular experiment coexisting fronts were observed to propagate at different speeds.

The orientation of banded localized deformation has received significant attention over the years and the present set of biaxial experiments offer a means for evaluating various propositions. The orientation of the bands is determined at the nucleation of transformation. In the uniaxial tension case, two bands initiate from the ends of the specimen and enter the FOV. Figure 7 shows an expanded view of the band at the lower end of the FOV. The inclination of the band to the axis of the tube, $\phi$, is taken to be that of the bisector of the edges of the inclined fingers. In this case $\phi=55.8^{\circ}$, a value that corresponds to inclinations measured in tension tests on NiTi strips (Shaw and Kyriakides, 1998) and steel strips that develop Lüders bands (Kyriakides and Miller, 2000; Hallai and Kyriakides; 2013).

A separate tensile experiment was performed that was monitored with an infrared camera in order to assess temperature changes induced by latent heats of the transformations. The specimen was coated with a matte black paint and DIC lights were not used to minimize IR noise and avoid any potential additional heating. The specimen was pulled at the same displacement rate as that used in the test in Figs. 3, with the net extension of the specimen monitored with a non-contacting laser extensometer. The stress-average strain response recorded has plateaus with very similar stress levels and extents, as are the deformation patterns that can be inferred from the thermographic record. The slow propagation of fronts coupled with relatively small 
temperature changes and the thin wall of the specimen results in a diffuse temperature profile. During the $\mathrm{A} \rightarrow \mathrm{M}$ transformation an increase of about $0.9^{\circ} \mathrm{C}$ was observed at front edges with a maximum temperature increase of $1.4^{\circ} \mathrm{C}$ at the coalescence of propagating fronts. The $\mathrm{M} \rightarrow \mathrm{A}$ transformation profile was again diffuse, although less so than that of the forward transformation, with front temperatures of $-0.7^{\circ} \mathrm{C}$ and a minimum of $-1.8^{\circ} \mathrm{C}$ at coalescence.

\section{b. Biaxial Experiment: $\sigma_{X}=0.375 \sigma_{\theta}$}

This is a biaxial experiment loaded under volume control as outlined in Section 2 and as such is representative of the rest of the biaxial experiments in this study. The water was pumped into the specimen at a rate of $0.02 \mathrm{ml} / \mathrm{min}$ and the induced radial stress history is shown in Fig. 8. During homogeneous deformation, this induced a hoop strain rate of $2.8 \times 10^{-6} \mathrm{~s}^{-1}$. Figure 9 shows the nominal stress-mean strain $(\sigma-\bar{\varepsilon})$ responses recorded in the axial and hoop directions. DIC images were taken at $6 \mathrm{~s}$ intervals and Fig. 10 shows 20 select sets of axial and hoop strain contours corresponding to the numbered bullets on the responses in Fig. 9. Figure 11 shows the corresponding histograms of hoop strain. Both responses trace closed hystereses, with $\sigma_{\theta}-\bar{\varepsilon}_{\theta}$ being significantly larger. Because $\alpha$ is relatively small, the axial strain is dominated by the Poisson's effect and as a result it is negative. The initial linear moduli of the responses of biaxial experiments are listed under $\sigma_{\theta} /\left.\varepsilon_{\theta}\right|_{A}$ and $\sigma_{X} /\left.\varepsilon_{X}\right|_{A}$ in Table 1. A gradual reduction in stiffness commences at a hoop stress of about $500 \mathrm{MPa}(72.5 \mathrm{ksi})$ presumably due to some transformation to $\mathrm{M}$, but deformation remains uniform at least up to $\sigma_{\theta}=542$ (image (1)). Subsequently, transformation to $\mathrm{M}$ accelerates forming gradual knees in the two responses that ease into nearly flat stress plateaus. At $\left(\sigma_{\theta}, \bar{\varepsilon}_{\theta}\right)=(549 \mathrm{MPa}, 0.78 \%)$ localized deformation in the form of a sharp tipped band has entered the FOV (seen in image (2) at $\sigma_{\theta}=556 \mathrm{MPa}$ ) at an inclination of $32.7^{\circ}$ to the axis of the tube. It is interesting to observe that, unlike the pure tension test, here nucleation occurs at gradually increasing stresses. Because of this rounding we have adopted two methods for establishing the nucleation of transformation. The first is based on the "inelastic"

work $\Delta W^{t}$, which is a measure of the nonlinearity of the response. The second is based on the intersection of two tangents at the knee of the response as shown in Fig. A1. The nucleation stresses $\left\{\sigma_{X N M}, \sigma_{\theta N M}\right\}$ listed in Table 1 are based on the second criterion. 
The more detailed record in the form of a video (s $\square \square \square \square \square \square \square \square$ alp_0.375) shows this initial band to develop into a multi-pronged one that by image (3) has evolved into a triangular island with a hoop strain of about $5 \%$ while the rest of the FOV remains relatively undeformed at the strain of the stress knee $(0.85 \%)$. While this island of transformed material grows, a second narrow spiral band of transformation develops from the top right with the same orientation as the initial band but propagating in the opposite sense as shown in image (4)

The two transformed zones grow and coalesce near the mid-height of the specimen at $\bar{\varepsilon}_{\theta} \approx 2.52 \%$. The coalescence leaves behind a diamond shaped island of A near mid-height (image (5). Subsequently, transformation switches to mainly broadening of the upper zone with less change in the lower one, followed by a new narrow band at the top, which can be seen fully developed in image 7 . Beyond point 9 , most of the remaining islands of A have transformed and the stress takes an upswing. Loading is terminated at point (10) at $\sigma_{\theta}=626 \mathrm{MPa}$. In Table 1 we report the extent of the transformation plateaus as $\Delta \varepsilon_{\theta M}^{t}$ and $\Delta \varepsilon_{x M}^{t}$. They represent the difference in strains at the beginning and end of the plateau established by the extrapolations shown in Fig. A1.

Unloading is conducted at the same flow rate and deformation is once more nearly uniform through point (11. However, some weak striped lower strain patterns near the middle of the specimen become discernible. An abrupt knee forms at $\sigma_{\theta} \approx 340 \mathrm{MPa}$ as $\mathrm{M} \rightarrow \mathrm{A}$ transformation initiates in the specimen. Three parallel narrow islands of transformed material appear and can be seen in the middle of image (12) at the location mentioned earlier. Clearly these are sites that were the last to transform to $\mathrm{M}$ and had required somewhat higher stress to complete transformation. It appears that on unloading they start to transform to $\mathrm{A}$ at a somewhat higher stress than the rest of the specimen. The stress stabilizes at about $335 \mathrm{MPa}$ and soon thereafter additional transformation fronts appear first at the top and then at the bottom of the FOV. These are also zones that transformed late to M. It is also interesting that all transformation zones and fronts have the same orientation, which also corresponds to what was observed during the $\mathrm{M}$ transformation. In image ${ }^{(13)}$ a triangular island of $\mathrm{A}$ has formed at the bottom and an approximately V-shaped one at the top. In images (15) and (16) these two zones have propagated towards the center with clear inclined fronts. Simultaneously the central striped islands of A have started growing so that by image (17) a single inclined zone of $\mathrm{M}$ remains. It continues to narrow 
until in image (19) at $\left(\sigma_{\theta}, \bar{\varepsilon}_{\theta}\right)=(322,0.62 \%)$ it has been reduced to a single narrow band. Transformation is completed when the hysteresis closes and further unloading follows the trajectory traced during loading.

One other variable that we will be tracking is the strain energy expended during the loadunload cycle, $W^{t}$. It represents the areas enclosed by the two hystereses, which in this case is $12.93 \mathrm{MPa}$ (Table 1).

The deformation histograms in Fig. 11 give an overall picture of the extent and evolution of inhomogeneous deformation in the FOV. In (1) and (2) the distribution is unimodal centered around $\bar{\varepsilon}_{\theta}$ of about $1 \%$, at the beginning of the stress plateau. Subsequently it becomes bimodal with a second peak centered about a strain of $5 \%$, that corresponds to nearly the end of the plateau. The larger strain peak gradually becomes the major mode during A-M transformation with the small strain peak disappearing in (9. The reverse transformation exhibits similar strain distributions following the reverse order.

In this experiment the temperature was monitored with an infrared camera. During A-M transformation, a temperature change of $1.2{ }^{\circ} \mathrm{C}$ was recorded at the edges of propagating fronts while a maximum temperature increase of $2.2{ }^{\circ} \mathrm{C}$ was recorded at the coalescences of transformation fronts. This temperature change may have contributed to the observed increase in the stresses, but is not large enough to justify the whole increase. During the reverse transformation, a diffuse cooling front of $-1.2{ }^{\circ} \mathrm{C}$ developed that initially followed the propagating fronts at the bottom and top of the FOV. From points (16) to (19) this temperature decrease spread to the whole central section.

In summary, for this stress ratio nucleation of $\mathrm{M}$ and $\mathrm{A}$ transformation resulted in less abrupt stress transitions, but their evolution led to inhomogeneous deformation.

\section{SUMMARY OF BIAXIAL EXPERIMENTS}

We now review results from the rest of the biaxial experiments concentrating on a select number of experiments whose behavior is representative of those of neighboring stress ratios. Along the way, we will be tracking some of the main parameters of the responses and listing them in Table 1. The trends of the key parameters in the biaxial stress space examined will be presented and discussed in Section 6. 
(a) $\alpha=0\left(\sigma_{x}=0\right)$

This is a uniaxial test in the hoop direction and as such serves as a complement to the uniaxial tension test described in Section 3a. The specimen was also loaded at a volume rate of $0.02 \mathrm{ml} / \mathrm{min}$ which induced a hoop strain rate of $4 \times 10^{-6} \mathrm{~s}^{-1}$ during the homogeneous parts of the deformation (details about the effect of the testing facility stiffness on the induced strain rate appear in Bechle, 2016). The end-cap effect was compensated by feedback producing a purely $\sigma_{\theta}$ stress state as shown in Fig. 8. The recorded $\sigma_{\theta}-\bar{\varepsilon}_{\theta}$ response is shown in Fig. 12a and accompanying sets of twelve hoop and axial strain contours in Fig. 12b (see movie alp_0) The load-unload cycle produced a closed hysteresis similar to that for $\sigma_{X}-\bar{\varepsilon}_{X}$ one in Fig. 9. The initial elastic modulus, $E_{A}$, is $66.8 \mathrm{GPa}$ and the Poisson's ratio, $v_{A}$, is 0.426 , both values close to those of the $\sigma_{X}$ test. A mild nonlinearity in the response is observed above $340 \mathrm{MPa}$ but in image (1), at $454 \mathrm{MPa}$, the FOV remains uniformly strained. At $458 \mathrm{MPa}$ the stiffness undergoes a rapid drop forming a knee and a single dextral (right-handed) helical band initiates on the lower LHS of the specimen. This band initiated close to the middle of the specimen outside the FOV. Table 1 reports the transformation stress $\left(\sigma_{\theta N M}\right)$ to be just under $470 \mathrm{MPa}$, which compares with the corresponding value of $459 \mathrm{MPa}$ for the axial tension test (Table 1). This band can be seen more developed with the hoop strain reaching more than 5\% in image (2) somewhat later. It has an inclination of $29.1^{\circ}$ to the axis of the tube, which is somewhat smaller than the complement of the $55.8^{\circ}$ angle of the initial band inclination under uniaxial tension (Table 1). With increased deformation the band broadens and a second band appears near the top end while the stress stabilizes. Image (3) shows the lower third of the specimen transformed, and the spiral band at the top to have grown significantly. In image (4) the transformed zone in the lower part of the specimen continued its growth and the helical band above it has broadened further while the transformed zone in the lower part of the specimen stress has gradually increased to $478 \mathrm{MPa}$. This increase may have been caused by a temperature increase of approximately $1.6^{\circ} \mathrm{C}$ resulting from the released latent heat (recorded with an infrared camera in a separate experiment under the same loading and environmental conditions). With additional deformation, the existing bands broaden and several secondary bands develop. Transformation is essentially completed by $\bar{\varepsilon}_{\theta} \approx$ $5.4 \%$ at a stress of $485 \mathrm{MPa}$, but small residual islands of A (e.g., image (5) transform at somewhat higher stress. Loading is terminated at (6) at $\left(\sigma_{\theta}, \bar{\varepsilon}_{\theta}\right)=(509 \mathrm{MPa}, 5.66 \%)$. 
Interestingly, the transformation strain, $\Delta \varepsilon_{\theta M}^{t}$, is $4.76 \%$, which is significantly lower than the corresponding value of $6.17 \%$ for the axial tension experiment. The corresponding axial strain is strictly due to the Poisson effect and consequently the contours show the same patterns.

During unloading, the specimen initially deforms homogeneously until the stress drops to $323 \mathrm{MPa}$ when a single dextral band of A appears near the center of the FOV. It can be seen somewhat later in image (8) together with a second band. The first band appears to have initiated from the site that transformed last during the forward transformation. A stress plateau develops during which bands of A grow until the whole specimen is back to A. Unlike loading, several multi-pronged patterns develop during unloading that can be seen in images (9) and (10). The last island to transform is a band that appears to be at the same location as the one that nucleated first during loading. As unloading progressed the temperature dropped by $-1.6{ }^{\circ} \mathrm{C}$ and on its completion the stress has dropped to $363 \mathrm{MPa}$. Beyond this point the specimen unloads with modulus $E_{A}$. The hysteresis energy $W^{t}=10.21 \mathrm{MPa}$, which compares with $13.49 \mathrm{MPa}$ for the axial tension test (Table 1). Since the transformation stresses in the two directions have similar values, this difference is primarily due to the smaller hoop direction transformation strains.

Our experimental setup facilitated the conduct of a number of experiments with negative values of $\alpha$. This was achieved by reducing the length of the test section in order to ensure that axial compression did not lead to buckling. We place these experiments in two groups with similar behavior.

(b) $-0.50 \leq \alpha \leq-0.25$

The first group involves experiments with stress ratios of $-0.25,-0.375$ and -0.50 . We briefly discuss results for $\sigma_{X}=-0.375 \sigma_{\theta}$, which are representative of all three. The length of the test section was reduced to $38 \mathrm{~mm}$ (1.5 in) and pressurization was conducted at a volume rate of $0.015 \mathrm{ml} / \mathrm{min}$. The induced radial path is shown in Fig. 8. The recorded stress-average strain responses are shown in Fig. 13a and sets of corresponding strain contours in Fig. 13b. The axial and hoop stress-average strain responses trace closed hystereses characterized by nearly flat stress plateaus and transformation leads to inhomogeneous deformation. A major difference from responses for positive $\alpha$, is that both the axial stress and strain are compressive. Transformation starts at $\left(\sigma_{X}, \sigma_{\theta}\right)_{N M}=(-155,416) \mathrm{MPa}$ as the two responses develop rather sharp knees. 
Simultaneously, a single sinistral (left handed) band of transformed material initiated from the upper gripped end of the specimen with an inclination of $30.5^{\circ}$ to the axis of the tube (see image (1). The band gradually broadens and lengthens covering first the upper part of the FOV (see image (2), but gradually propagating towards the lower end as shown in images (4) and (5) Interestingly, in this case transformation evolved through this single band. Residual islands of A at the top and bottom transform when $\sigma_{\theta}$ is increased to $458 \mathrm{MPa}$ (image (6)).

On unloading, the specimen deforms uniformly down to $\sigma_{\theta}=243 \mathrm{MPa}$ when transformation to A initiates from the zones that transformed last during loading in the upper and lower corners of the FOV (see image (8). The hoop and axial stresses trace plateaus at approximately $234 \mathrm{MPa}$ and $-87 \mathrm{MPa}$ respectively while inclined transformation zones propagate from the upper and lower corners inwards. In essence, the transformation bands follow a nearly reverse evolution to that followed during loading. Both hystereses close and further unloading follows the loading responses. The hysteresis energy is now $10.27 \mathrm{MPa}$.

Overall, the results for $\alpha$ of -0.25 and -0.50 exhibit similar trends. Their responses are similar to those of $\alpha=-0.375$ with the measurables listed in Table 1. As the axial stress becomes more compressive, the hoop transformation stress decreases and $\Delta \varepsilon_{X}^{t}$ increases. The hysteresis energy for $\alpha=-0.25$ is nearly the same as for 0 , but increases for the two lower values of $\alpha$ (see Section 6). For -0.50 , transformation induced deformation is mainly through the evolution of a single band, similar to what is observed in Fig. $13 \mathrm{~b}$ for -0.375 . By contrast, -0.25 exhibited more multi-helical localization patterns that resemble those observed for $\alpha=0$.

(c) $-1.0 \leq \alpha \leq-0.75$

The second group of experiments with compressive axial stresses includes stress ratios of $-0.75,-0.88$ and -1.00 . The length of the test section was reduced to $32 \mathrm{~mm}(1.25 \mathrm{in})$ and pressurization was conducted at a volume rate of $0.015 \mathrm{ml} / \mathrm{min}$. We discuss results from $\sigma_{X}=-0.75 \sigma_{\theta}$ that are representative of the three in this group. The induced radial path is shown in Fig. 8 (a brief deviation observed in this radial path is due to temporary loss of feedback control during unloading). The recorded stress-average strain responses are shown in Fig. 14a and sets of corresponding strain contours in Fig. $14 \mathrm{~b}$ - see movie alp_- 0.75 (note that because of the shorter test section, the field of view has been reduced to $16.4 \times 6.5 \mathrm{~mm}$ ). The nucleation of $\mathrm{M}$ 
during loading and A during unloading induce quite sharp stress knees. In fact, a small stress peak develops at the nucleation of $\mathrm{M}$. Transformation progresses by inhomogeneous deformation reflected in both strain contours. It takes the form of multi-pronged sinistral helixes of $\mathrm{M}$ that appear nearly simultaneously both at the lower and upper ends of the FOV (image (2)). Interestingly, the lower cluster has more prongs than the upper one, but both sets of helixes are inclined at about $38^{\circ}$ to the tube axis. This inclination is somewhat larger than the angles measured for single nucleation bands from the other negative stress ratio experiments (Table 1). In images (3) and (4) the helixes grow gradually transforming the rest of the test section with the stresses remaining essentially unchanged. The helixes meet in image (5) and the stresses start to increase indicating the completion of the unstable part of the transformation.

During unloading, the specimen deforms uniformly down to $\left(\sigma_{X}, \sigma_{\theta}\right)$ of about (148,199) MPa when transformation to A initiates at the top end with multi-helical bands of both chirality (see image (8). This deformation pattern propagates downwards (image (9) with the stresses remaining essentially unchanged. The propagation continues until $\left(\bar{\varepsilon}_{X}, \bar{\varepsilon}_{\theta}\right) \approx(-1.85,3.18) \%$ when the sinistral bands decay and simultaneously dextral bands initiate from the bottom of the FOV (seen somewhat later in image (10). This switch appears to be necessary for compatibility between the top and bottom helixes. Further transformation is by upward propagation of the lower helixes and is completed a little after image (11) with the stresses dropping to $(-143,192) \mathrm{MPa}$. The two hystereses have closed and further unloading is by uniform deformation of A. The hysteresis energy $W^{t}$ is $10.52 \mathrm{MPa}$ a value that is in line with those of the other negative stress ratio experiments.

The results for $\alpha$ of -0.88 and -1.0 have the same trends. The transformation stresses continue to drop as $\alpha$ becomes more negative. Transformation induces inhomogeneous deformation with the stresses remaining essentially constant both during loading and unloading. For $\alpha=-0.88$, A transforms by multi-pronged sinistral helixes that emanate from the top and bottom ends and propagate towards the middle. In the case of -1.0 the multi-pronged helixes are of both chiralities. On unloading, M transforms mainly with multi-pronged sinistral helixes for 0.88 but for -1.0 single inclined bands develop like those reported for larger $\alpha$. It is worth pointing out that multi-pronged helical bands with dual chirality appear to be more common for shorter specimens as a means of staying straight dictated by the length constraint (e.g., see Feng 
and Sun [2006], Reedlunn et al., 2014 for tubes and Shaw and Kyriakides, 1998 for strips transforming under axial tension). The extents of the hoop strain plateaus decrease and those of axial strain increase somewhat as $\alpha$ becomes more negative. The transformation energy in these two cases is at a similar level as that of the other negative stress ratios.

(d) $0<\alpha \leq 0.50$

Experiments with lower positive values of $\alpha$ were conducted for $0.05,0.25,0.375$ and 0.50 . Overall the behavior recorded was similar to that of $\alpha=0.375$ outlined above. The trends observed and highlights can be summarized as follows. The onset of $\mathrm{M}$ transformation is less abrupt than those of the two uniaxial loading cases, inducing a somewhat rounded stress knee. Transformations leads to inhomogeneous deformation in the form of inclined bands that start with an inclination that hovers around $30^{\circ}$ to the axis of the tube, but $\phi$ drops to about $25^{\circ}$ for $\alpha$ $=0.50$. As the loading continues, the higher deformation bands grow, gradually covering the whole specimen while the stress remains nearly constant. Both nucleation stresses, $\sigma_{X N M}$ and $\sigma_{\theta N M}$, increase with $\alpha$ (Table 1). The hoop transformation strain, $\Delta \varepsilon_{\theta M}^{t}$, remains nearly unchanged while $\Delta \varepsilon_{X M}^{t}$ gradually decreases as $\alpha$ increases.

On unloading, the specimens initially deform homogeneously. Transformation back to A initiates with rounded transitions to relatively flat stress plateaus. The transformation stresses $\left(\sigma_{x N A}, \sigma_{\theta N A}\right)$ increase with $\alpha$. During the lower stress plateau the deformation is inhomogeneous with its evolution following approximately the reverse order to that followed during loading. The hysteresis energy, $W^{t}$, increases with $\alpha$ reaching $12.93 \mathrm{MPa}$ for 0.375 but drops to $12.37 \mathrm{MPa}$ for 0.5 (Table 1).

(e) $\alpha=0.60$

The responses and the deformation images for $\sigma_{X}=0.60 \sigma_{\theta}$ shown in Fig. 15 appear to set different trends than those of the lower values of $\alpha$ reported thus far. The transition "knees" in the two responses are more gradual and the two stresses monotonically increase/decrease during transformation. Following the trend set thus far, transformation nucleates at even higher stresses $\left(\sigma_{X}, \sigma_{\theta}\right)_{N M}=(361,596) \mathrm{MPa}$. Furthermore, the first signs of inhomogeneous deformation are 
the form of relatively weak axial bands such as those seen in image (2) and more intense in image (3) Therefore, the band inclination $\phi$ is reported as $0^{\circ}$ in Table 1 . It is noteworthy that these initial bands are not registered in the corresponding axial strain contours. Inclined bands of higher strain emanate from the top and bottom of the FOV around a hoop stress of $606 \mathrm{MPa}$, and a strain of $1.85 \%$ just before image (4). In this image a single dextral narrow band has also developed near the lower end. The inclined bands propagate towards the center (image (5) where they meet forming a diamond shaped island of relatively undeformed material that can be seen in image (6). Transformation is completed around $\left(\sigma_{\theta}, \bar{\varepsilon}_{\theta}\right)=(629 \mathrm{MPa}, 4.74 \%)$ but loading continued to a hoop stress of $673 \mathrm{MPa}$. In the process the axial response traces a higher stress than in lower stress ratios (due to the higher $\alpha$ ) but a smaller strain.

On unloading from (7) the specimen deforms homogeneously down to $\left(\sigma_{\theta}, \bar{\varepsilon}_{\theta}\right)=$ (390 MPa, 4.05\%) just beyond point (8). An island of lower strain has nucleated at the site of the termination of forward transformation. This island develops into two sinistral inclined bands that can be seen in image (9. The bands broaden and lengthen as can be seen in image (10. In image (11) most of the FOV has been transformed to a hoop strain of about $0.42 \%$ but a weak narrow band of higher strain remains in the center of the FOV. In image (12) this band is the only zone of higher strain that remains and is reminiscent of a similar band in image (2) during loading. This band is seen to be disappearing in image (13) very close to the point where the response rejoins the initial linear modulus of A. The axial deformation reflects the hoop strain patterns except that the weak longitudinal bands at the end are not registered.

In summary, the $M$ nucleation stresses have increased for this stress ratio as have the stresses at the completion of transformation with the difference between nucleation and completion increasing over those of smaller $\alpha$ (due to monotonic increase in stresses during transformation). The transformation is completed in smaller strains and $W^{t}$ is smaller. The initial angle of transformation is essentially $0^{\circ}$.

(f) $\alpha=0.85$

We now consider results for $\alpha=0.85$ (Fig. 16, see movie alp_0.85), which turns out to be very similar to those of 0.75 . Transformation nucleates at higher stresses than in smaller values of $\alpha$ forming a gradual transition knee at $\left(\sigma_{X}, \sigma_{\theta}\right)_{N M}=(553,614) \mathrm{MPa}$. Transformation takes 
place with increasing stress (Table 1) and is completed in smaller strains than previous stress ratios. Localized deformation in the form of a weak axial band is seen in the hoop contour in image (2) during the stress knee. It grows axially as seen in image (3) and progressively consumes the entire FOV as seen in images (4), (5) and (6). Transformation appears completed by $\left(\sigma_{\theta}, \bar{\varepsilon}_{\theta}\right) \approx$ (685 MPa, 3.53\%), but the stress was taken to $735 \mathrm{MPa}$ and hoop strain of $3.89 \%$ before commencing unloading (7). As pointed out earlier, the axial strain has been decreasing as $\alpha$ increased. For this particular stress ratio, no axial hysteresis is traced and, in tandem, the hoop strain localization does not register in the axial strain contours. Reducing the DIC scale to 0 to $1.0 \%$ confirms this lack of localization. The Poisson effect of the dominant hoop transformation strain appears to be a near perfect complement to the corresponding axial transformation strain.

On unloading, transformation to $\mathrm{A}$ commences at approximately $\left(\sigma_{\theta}, \bar{\varepsilon}_{\theta}\right) \approx$ (469 MPa, 3.13\%). It takes the form of axial bands of lower strain that emanate from the bottom of the FOV. In fact, it appears that A nucleates at the site that transformed last during loading (compare images (6) and (9). The lower strain zone propagates upwards following essentially the reverse path to that followed during loading. Soon thereafter the specimen unloads with the initial linear loading modulus and the hysteresis closes. As was the case for loading, no change in the axial strain is registered in the unloading strain contours.

In summary, the nucleation stresses have increased and more pronounced hardening is observed during forward and reverse transformation. Transformation involves inhomogeneous deformation with a $0^{\circ}$ orientation. It is completed in smaller hoop strain and no change in the corresponding axial strain. In addition, the hysteresis energy, $W^{t}$, has reduced from the local maximum value that occurred for $\alpha=0.375$.

The observations made for $\alpha=0.85$ hold also for 0.75 except that for this case a small $\sigma_{X}-\bar{\varepsilon}_{X}$ hysteresis with negative strain was recorded.

(g) $1.0 \leq \alpha \leq 1.20$

Four sets of results near the equibiaxial stress ratio are discussed together, $\alpha=1.0,1.07$, 1.15 and 1.20, because of common features exhibited by their results. In the case of $\alpha=1.0$ (see Fig. 17, see movie alp_1.0) the transformation stresses have continued to increase while the axial strain is now positive. However, the extent of the dominant hoop transformation strain has 
decreased and consequently so has the transformation energy $W^{t}$. It is noteworthy that despite the equibiaxial state of stress, the axial transformation strain is significantly smaller than that in the hoop direction, $\left(\Delta \varepsilon_{X}^{t}, \Delta \varepsilon_{\theta}^{t}\right)=(0.94,2.18) \%$, another sign of the anisotropy exhibited by the transformation strain pointed out earlier from the results of the two uniaxial stress experiments. Transformation induces even more gradual transition knees and progresses with even steeper stresses increases with $\sigma_{\theta}$ for example increasing by $101 \mathrm{MPa}$ between nucleation of $\mathrm{M}$ and termination of transformation. The more dominant hoop strain contours exhibit very mild inhomogeneity with an axial orientation that broadens and spreads to the entire FOV (see images (3)-(5). In the axial strain contours inhomogeneity is diffuse and a preferred orientation cannot be discerned. Reducing the DIC strain scale to $0-1.75 \%$ reveals weak features with diffuse strain gradients that are on the order of $\Delta \varepsilon_{X}^{t}$. Grolleau et al. [2011] in a hydraulic bulge test on a circular NiTi sheet noted a hardening behavior and no localization at the apex.

In the case of $\alpha=1.07$ (Fig. 18) the axial response becomes dominant for the first time. Transformation induces gradual transition knees and monotonically increasing stresses. The results indicate that the responses develop equal hoop and axial transformation strains for a value of $\alpha$ between 1.0 and 1.07. The transformation strains remain relatively small as does $W^{t}$. The axial strain contours appear to be homogenous during both loading and unloading, which is confirmed by finer DIC scales. The hoop strain contours exhibit a mild inhomogeneity with a predominantly axial orientation. An axial feature first seen in image (2) gradually spreads to the whole FOV. In this case loading may have been terminated before transformation to $\mathrm{M}$ was completed. Thus the reported $\left(\sigma_{X}, \sigma_{\theta}\right)_{M}$ values reported in Table 1 originated from a second experiment with the same stress ratio that was taken to higher stresses.

The results for $\alpha=1.15$ are similar to those of 1.07 and will not be shown. The responses remain monotonic, the size of the hoop hysteresis is reduced further, and $W^{t}$ remains relatively low. The axial strain is essentially homogeneous with the contours exhibiting diffuse patterns with no identifiable preferred orientation. The hoop strain contours display a mild inhomogeneity with predominantly axial orientation.

Figure 19 shows the results for $\alpha=1.20$ where the hoop hysteresis has almost disappeared-see movie alp_1.20 (similar to axial response for $\alpha=0.85$ ). The axial response 
maintains its monotonic nature with the axial transformation strain recording an increase. The transformation stresses remain relatively high, and $W^{t}$ registers an increase over the previous three stress ratios. The axial deformation is essentially homogenous exhibiting very mild features oriented in the transverse direction. The hoop strain is very small and homogeneous except for a suspect axial region on the left edge of the FOV. At a reduced strain scale of $0-1.0 \%$ the hoop contours confirmed homogeneity outside this edge region prompting the conjecture that it may be an edge effect caused by the small values of strain involved.

In summary, all results close to the equibiaxial stress state exhibit responses with monotonically increasing stresses and as a result a significant difference is registered between the values at the onset and completion of transformation. The transformation strains are both positive and at their smallest achieving equal values around a stress ratio of about 1.05. The hysteretic energy recorded is at its lowest and relatively constant. Deformation is essentially homogeneous with mild features appearing for some cases that are mainly in the transverse direction for the axial strain and in the axial direction for the hoop strain.

(h) $\alpha=1.26$ and 1.30

The two stress ratios of 1.26 and 1.30 represent the transition to axially dominant deformations and to increasingly more inhomogeneous deformations. Furthermore, the hoop hysteresis has now switched to negative strain. Figure 20 shows the results of 1.30 , which are representative of both cases. Figure 20a shows that transformation continues to lead to rounded transition knees followed by rising stress responses. The transformation stresses $\left(\sigma_{X}, \sigma_{\theta}\right)_{N M}=$ $(669,525) \mathrm{MPa}$ are of the same order of magnitude as those in the equibiaxial neighborhood (Table 1). Deformation remains essentially homogeneous (see image (2) until the axial strain reaches about $1.35 \%$ when mild inhomogeneity is observed at the top and bottom of the FOV. It consists of patterns nearly orthogonal to the tube axis at the top and slightly inclined ones at the bottom. They can be seen in a more advanced state of development in image (3). The mild inhomogeneity is not registered in the hoop strain profiles presumably because their magnitude is significantly smaller. As the loading progresses, the transformation patterns become stronger with islands forming at the top and bottom (see image (4)), gradually spreading towards the center (see image (5)). The patterns remain primarily in the transverse direction, but several ones with slight inclinations can be also seen in images (3) and (4). No inhomogeneity is registered in the 
hoop strain profiles throughout the loading in the strain scale of Fig. 20. Reducing the DIC scale to $-1.0 \%-1.0 \%$ reveals very weak features on the order of the DIC strain resolution. Inhomogeneous deformation is completed by $\left(\sigma_{X}, \bar{\varepsilon}_{X}\right) \approx(710 \mathrm{MPa}, 4.32 \%)$ when the stress develops an increasing trend and loading is terminated. This experiment was also monitored with an infrared camera, which recorded a temperature increase of $1.6^{\circ} \mathrm{C}$ at active transformation fronts.

Upon unloading, as transformation to $\mathrm{M}$ starts, a gradual stress knee is traced. The transformation stresses based on the tangent constructions (Fig. A1) are $\left(\sigma_{x}, \sigma_{\theta}\right)_{N A}=(415,325)$ MPa. First signs of inhomogeneous deformation appear at $\left(\sigma_{x}, \bar{\varepsilon}_{X}\right) \approx(413 \mathrm{MPa}, 3.58 \%)$ when weak, nearly transverse patterns appear at the sites last transformed during loading. They can be seen somewhat later in image (8). As unloading progresses, the patterns strengthen and spread throughout the FOV as observed in images (9) and (10. The patterns are mostly in the transverse direction, but some have small inclinations. As was the case during loading, the hoop strain contours remain homogeneous. At the end of transformation at point ${ }^{12}$, the axial stress dropped down to $369 \mathrm{MPa}$. During the evolution of transformation the temperature of active fronts dropped by $0.7^{\circ} \mathrm{C}$.

Overall for these two stress ratios the transformation strains have increased over those near the equibiaxial stress ratios and the hysteresis energies are also on a rising trend. The band initiation angles have been registered as $90^{\circ}$.

(i) $1.52 \leq \alpha \leq 8.0$

We now report results from a group of five radial paths with stress ratios that range from 1.52 to 8.0. They share many features that will be outlined through the representative case of $\alpha=$ 4.0. Figure 21 shows the recorded stress-average strain responses and the corresponding strain contours from DIC (see movie alp_4.0). The responses trace closed hystereses with rather sharp transition stress knees followed by relatively flat plateaus for both loading and unloading. The transformation starts at $\left(\sigma_{X}, \sigma_{\theta}\right)_{N M}=(541,138) \mathrm{MPa}$ and leads immediately to inhomogeneous deformation. In image (2) just after the onset of transformation, a sinistral helical band of higher strain starts at the upper end, and wraps around the specimen with its sharp front appearing below. The axial strain in the band is about 7\% (corresponding to the strain at the end of the 
stress plateau) while the rest of the specimen remains approximately at $0.84 \%$ (strain at nucleation of $\mathrm{M}$ ). The inclination of the front to the axis of the tube is about $60^{\circ}$. Image (3) shows the helical band to have propagated one more time around the specimen, the front reaching the lower third of the length of the FOV while behind it the band has widened. In image (4) the end of the band has widened further and its front has reached the lower edge of the FOV. Subsequently, transformation takes place by widening of the now stationary band as seen in image (5. In image (6) a single sliver of A remains which, soon thereafter, transforms leading to the termination of the stress plateau and the observed upswing in the stress (point (7)). It is interesting that the axial stress at the end of the plateau has reached $553 \mathrm{MPa}$, an increase of $12 \mathrm{MPa}$ over the stress at the nucleation of $\mathrm{M}$. The hoop strain contours are negative replicates of the axial strain but at much smaller levels.

On unloading from (7), deformation remains homogeneous until point (8) at $\left(\sigma_{X}, \bar{\varepsilon}_{X}\right)=$ (256 MPa, 6.12\%). A sinistral multi-helical front of A enters the FOV at the lower end just before image (9. It propagates upwards developing into a front with helixes of both chiralities. In image (10) a second multi-helical front enters the FOV from the top. The two fronts of A propagate towards each other and in image (12) they have evolved into "chevron" like patterns where transformation takes place along both characteristics. In image ${ }^{13}$, but for a small sliver, the specimen has transformed back to A while the stress drops to $221 \mathrm{MPa}$.

For stress ratios of 2.0 and higher the induced change in the specimen volume becomes increasingly smaller. Due to experimental constraints the flow rate was kept at $0.02 \mathrm{ml} / \mathrm{min}$ thus the induced strain rate increased. This meant that radiation and conduction could not dissipate the latent heat of transformation fast enough causing small increases in temperature during loading and decreases during unloading. The changes in temperature were larger than the approximately $\pm 1{ }^{\circ} \mathrm{C}$ value reported for experiments with $\alpha<1.3$. Thus for example, in the case of $\alpha=2.0$ a temperature increase of $2.6{ }^{\circ} \mathrm{C}$ was recorded at the propagating fronts of transformation during loading and a decrease of $2.1{ }^{\circ} \mathrm{C}$ during unloading. In both cases the temperature changes nearly doubled in the latter parts of the stress plateaus when transformation fronts interacted with each other and eventually coalesced. The corresponding values for the 8.0 stress ratio were $3.0^{\circ} \mathrm{C}$ and $-2.8^{\circ} \mathrm{C}$ at discrete transformation fronts and again nearly double in the latter parts of the stress plateaus. Although thermal imaging was not performed for 4.0, we expect the temperature changes to be of the same order as these two sets of values. Since the 
transformation stress is temperature dependent, we consider the small increase in the stress during loading and decrease during unloading in Fig. 21 to be mainly caused by thermal heating and cooling.

The results for stress ratios of 1.52, 1.79, 2.0 and 8.0 exhibit broadly similar behaviors. As $\alpha$ increases, transformation results in more abrupt stress knees and both transformation stresses progressively decrease. It is important to point out that the values of the A nucleation stresses are not affected by latent heat effects so they correspond to $23{ }^{\circ} \mathrm{C}$. Transformation progresses with the stress remaining essentially constant. Small increases in stress during $\mathrm{A} \rightarrow \mathrm{M}$ and decreases during $\mathrm{M} \rightarrow \mathrm{A}$ transformation appear to be mainly due to mild heating and cooling resulting from latent heats. In all five cases transformation resulted in inhomogeneous deformation in the form of inclined bands of transformed material. The initial inclination of the bands was about $60^{\circ}$ to the axis of the tube dropping to approximately $55^{\circ}$ for uniaxial tension. Multi-helical transformation fronts were observed for $\alpha$ of 4.0 and 8.0 during unloading. The strains at the termination of transformation increase with $\alpha$ until $\alpha=2.0$ at which point the strains remained similar to the values recorded for uniaxial tension. The hysteretic energy exhibits the increasing trend observed for $\alpha$ of 1.0 and higher reaching a maximum value at 2.0. The energy exhibits a deceasing trend for higher stress ratios.

\section{BAND ANGLE}

For a significant number of the stress ratios of the biaxial experiments conducted, the nucleation of $\mathrm{M}$ consistently resulted in the formation of a narrow band of higher strain material. The band is inclined to the axis of the tube by an angle $\phi$. Similar bands of discontinuous deformation are commonly observed at the onset of Lüders banding in mild steels and in other materials (e.g., Kyriakides and Miller, 2000; Hallai and Kyriakides, 2013). In a plane stress setting, Hill [1952] examined the conditions that will allow the perfectly plastic material inside a band to develop velocity $\underline{\dot{u}}$ that is different from that of the material on either side of it as shown in Fig. 22 (see also Bijlaard, 1940). This velocity vector is at an angle $\psi$ to the band. The constraint provided by the material on either side of the band dictates that the strain rate induced by $\underline{\dot{u}}$ along the band is zero (direction $t$ ). Using the kinematics of the band, the angle $\psi$ is shown to be related to the principal strain rates inside it, $\dot{\varepsilon}_{1}$ and $\dot{\varepsilon}_{2}$, through 


$$
\sin \psi=\frac{\dot{\varepsilon}_{1}+\dot{\varepsilon}_{2}}{\dot{\varepsilon}_{1}-\dot{\varepsilon}_{2}} .
$$

Adopting an appropriate yield function, $\Phi\left(\sigma_{1}, \sigma_{2}\right)=$ Const., and an associative flow rule, the angle $\psi$ is related to the principal stresses through

$$
\sin \psi=\frac{\left(\partial \Phi / \partial \sigma_{1}+\partial \Phi / \partial \sigma_{2}\right)}{\left(\partial \Phi / \partial \sigma_{1}-\partial \Phi / \partial \sigma_{2}\right)}
$$

Thus, for isotopic yielding, uniaxial tension leads to $\dot{\varepsilon}_{1}=-\dot{2} \varepsilon_{2}$

and

$$
\psi=\sin ^{-1}(1 / 3)=19.47^{\circ} \text { and } \phi=\frac{\pi}{4}+\frac{\psi}{2}=54.74^{\circ} .
$$

Although transformation to $M$ is not strictly incompressible, this value of $\phi$ is close to that measured in the uniaxial tension experiment $\left(\sigma_{\theta}=0\right.$ in Table 1) on NiTi tubes (see similar results on NiTi strips in Shaw and Kyriakides, 1998).

Motivated by this success we explore what angle this simple criterion predicts for the biaxial experiments presented using the anisotropic transformation function $\Phi$ (CPB06) calibrated to the $\mathrm{M}$ nucleation stresses $\left(\sigma_{X}, \sigma_{\theta}\right)_{N M}$ as outlined in Appendix B using an exponent of 6. The resultant transformation surface is plotted in the $\sigma_{X}-\sigma_{\theta}$ plane in Fig. 23 together with the measured transformation stresses. The von Mises (vM) surface is included in the figure for comparison. The CPB06 surface develops the elongated trend exhibited by the data for $0.5<\alpha<2.0$ and captures the tension compression asymmetry, albeit with the limited available compressive data. By contrast, the vM surface is seen to deviate significantly from the measured transformation stresses.

The CPB06 transformation surface was used in Eq. (5) to develop predictions for the band angles, $\phi$. The predicted values of $\phi$ are plotted against the stress ratio angle $\tan ^{-1} \alpha$ in Fig. 24. Included for comparison are corresponding angles predicted if the transformation surface $\Phi$ is represented by the von Mises function. The predictions based on CPB06 are seen to follow the trend of the angles measured in the experiments reasonably well for the entire range of stress ratios that develop localized bands. For stress ratios $0.780 \leq \alpha \leq 1.412$ Eq. (5) has no solution, a result that agrees better with the observed behavior than the vM bounds of $0.5 \leq \alpha \leq 2.0$. The 
lack of prediction accuracy by the $\mathrm{vM}$ function is directly related to its inaccurate fit of the transformation stress surface.

\section{DISCUSSION OF RESULTS}

We now take an overall view of the experimental results and try to establish trends. Figures 25 and 26 show selected $\sigma_{X}-\bar{\varepsilon}_{X}$ and $\sigma_{\theta}-\bar{\varepsilon}_{\theta}$ responses for $\alpha \leq 1.0$ and $\alpha \geq 1.0$ respectively. For all stress ratios transformation resulted in stress-strain hystereses that closed on unloading. In other words, in this part of the $\sigma_{X}-\sigma_{\theta}$ space the material continues to exhibit the pseudoelastic behavior previously observed under uniaxial tension. The A phase was found to be isotropic linearly elastic with modulus $E_{A}=66.3 \mathrm{GPa}$ and Poisson's ratio $v_{A}=0.425$ (see Appendix C). For stress ratios ranging from -1.0 to 1.0 the hoop hysteresis dominates. As $\alpha$ is increased from -1.0, the transformation stresses gradually increase while the dominant hoop strain decreases. For $\alpha \leq 0.60, \mathrm{M}$ nucleates mostly as a narrow helical band of higher strain accompanied by a loss in stiffness in the form of a sharp stress knee. Transformation spreads most often through axial propagation and broadening of such bands with the stress remaining essentially constant. The deformation inside the bands typically corresponds to that at the end of the stress plateau while the rest of the specimen, still in the austenitic phase, is at the strain that $\mathrm{M}$ first nucleated at the beginning of the plateau.

On unloading, the martensitic phase initially deforms homogeneously. When the stress drops sufficiently, A nucleates forming again helical or multi-helical bands. A lower stress plateau is traced during which the bands propagate and broaden. In several cases the evolution of deformation follows the reverse order to that followed during loading. However, the lower stress of this transformation, coupled with structural effects, lead to other deformation patterns like multi-helical fronts for some cases.

The axial and hoop stress at the "nucleation" and "completion" of $\mathrm{M}$ and $\mathrm{A}$ transformations are plotted against each other in Fig. 27. Each pair of stresses was established by the "tangent" construction method defined in Fig. A1. The stresses corresponding to nucleation of M are fitted with the CPB06 "yield" surface outlined in Appendix B. The actual values and the fit are very similar to those reported in Fig. 23. For completeness, the other three sets of data are fitted in a similar fashion and are included in the figure. Since for $-1.0 \leq \alpha \leq 0.5$ transformation 
occurred with small increase in stress, the nucleation and completion stresses are very close to each other.

Figure 28a shows axial vs. hoop strain plots at the nucleation and "completion" of $\mathrm{M}$ transformation from the 26 radial experiments conducted. It is interesting to observe that the extent of strain induced by the transformation of $\mathrm{M}$ starts with large values for the negative and small values of $\alpha$ and decreases as the biaxial stress state is approached.

As $\alpha$ increases from 0.75 to 1.0 , the transformation stresses increase while the transformation strains decrease. The sharp transformation stress knee is replaced by a gradual reduction in modulus followed by hardening behavior until the completion of M (Fig. 25). Furthermore, transformation deformation is now mostly homogeneous with weak, mainly axial, patterns developing in the dominant hoop strain. As a consequence of the exhibited hardening, the stresses at the nucleation and completion of $\mathrm{M}$ in Fig. 27 are separated. For $\alpha=0.85$, the axial response did not develop a hysteresis and for higher values of $\alpha$ the axial hystereses have positive strain values (Figs. 25 and 28a). It is worth noting that at the equibiaxial stress state, the induced transformation strain is at its smallest. Furthermore, the axial strain is smaller than the hoop value, a sign of strain anisotropy.

Unloading in this stress ratio regime follows the same trends as loading. Nucleation of A is through a rounded stress transition knee followed by monotonically decreasing responses and mainly homogeneous deformation. Once more, the surfaces corresponding to nucleation and completion of A in Fig. 27 are separated. The associated transformation strains plotted in Fig. $28 \mathrm{~b}$ follow a similar trend as those of the forward transformation but have smaller values.

Figure 26 shows select responses for $\alpha \geq 1.0$. Once again, transformation results in fully recoverable deformation with all hystereses closing. For lower values of $\alpha$, in the neighborhood of 1.0, the responses are similar to those just outlined. Nucleation is associated with rounded stress transitions followed by hardening behavior for both loading and unloading. The axial and hoop transformation strains become equal at a stress ratio of about 1.05, but their values remain at their lowest level (Fig. 28a). Transformation develops essentially homogeneously with very weak, now in the transverse direction, patterns starting to appear in the axial strain at $\alpha=1.20$ and more prominently at 1.30 . For 1.20 the hoop response traces essentially no hysteresis and appears to be the dual to the disappearance of the axial hysteresis for $\alpha=0.85$. 
As the stress ratio increases beyond 1.30, transformation nucleates with increasingly sharper stress knees and its evolution occurs with minimal change in stress. The transformation is once more strongly inhomogeneous, developing helical bands of transformed material that propagate and broaden. Similar behavior is observed during the transformation to A on unloading, but the unloading patterns are often different from those developed during loading. As illustrated by the results in Fig. 28, the transformation strains increase with $\alpha$. Thus for the pure axial tension case, the $\mathrm{M}$ transformation strain reaches a value of about $6.9 \%$ which compares to $5.4 \%$ developed for the hoop uniaxial tension test $(\alpha=0)$. This transformation strain anisotropy occurs even though the transformation stresses in the two directions have very similar values.

The energy dissipated by transformation, $W^{t}$, plotted against the angle $\tan ^{-1} \alpha$ in Fig. 29 provides another global interpretation of NiTi behavior under biaxial stress states. For the negative values of $\alpha$, up to the pure hoop stress case, the energy remains nearly constant. It increases somewhat for small values of $\alpha$ reaching a maximum at 0.375 , and then drops to a minimum in the neighborhood of $\alpha=1.0$. The energy then increases significantly to a maximum value at $\alpha=2.0$ and drops again for larger stress ratios. Since the stresses at the beginning and end of the two transformations are seen in Fig. 27 to trace nearly symmetric loci, the large difference in $W^{t}$ between $\alpha<1.0$ and $\alpha>1.0$ is another symptom of the anisotropy in the transformation strains.

In many of the presented biaxial stress experiments, transformation nucleated by the sudden appearance of a narrow band of higher strain. Hill's [1952] condition for such discontinuities to develop in a perfectly plastic material was used to establish estimates of the band inclination for different values of $\alpha$. This was achieved by fitting the measured $\mathrm{M}$ nucleation stresses with the anisotropic yield function of Cazacu et al. [2006] that also allows for the tension/compression asymmetry exhibited by the NiTi tubes analyzed. The band angle dependence on $\alpha$ produced by this idealized model was found to yield results that follow the measured inclinations quite well, including the lack of solution for a relatively narrow range of stress ratios around the equibiaxial stress state. 


\section{CONCLUDING REMARKS}

We have investigated the solid-state phase transformation behavior of NiTi in the pseudoelastic temperature regime under biaxial stress states. NiTi tubes with diameters of 6.35 $\mathrm{mm}$, initially in the austenitic phase, were loaded under radial stress paths in the $\sigma_{X}-\sigma_{\theta}$ plane. Results for 26 biaxiality stress ratios of $-1.0 \leq \alpha \leq \infty$ have been reported. In each case the specimen was loaded up to the "completion" of martensitic transformation and unloaded. The use of stereo DIC for monitoring of the deformation in the test section enabled quantitative tracking of the evolution and degree of inhomogeneity associated with the transformation from A to $\mathrm{M}$ during loading and from $\mathrm{M}$ to A during unloading.

The most striking result is that outside of a narrow region spanning equibiaxial tension, transformation leads to localized helical deformation bands with inclinations that dependent on the stress ratio. During transformation the stresses remain nearly constant. In the vicinity of equibiaxial tension, the material hardens and deformation is homogeneous. The transformation stresses exhibited only mild anisotropy, but trace a surface that is elongated along the equibiaxial direction and deviates significantly from a von Mises representation. The transformation stress locus generated was well fitted using the anisotropic yield function of Cazacu et al. [2006] that also captures the tension/compression asymmetry exhibited by the tubes used in the experiments. A more complete representation will have to await experiments that complement the stress states covered. By contrast, the transformation strains exhibit significant anisotropy between axial and hoop dominant stress paths. Moreover, strains around the equibiaxial stress state, where material hardening and homogeneous deformation was observed, were significantly smaller than in the rest of the stress space. The strain anisotropy had a corresponding influence on the energy dissipated during transformation with axial dominant stress paths dissipating significantly more energy than hoop dominant ones, and less dissipation in the neighborhood of equibiaxial stress. These are features that constitutive models for NiTi must be able to reproduce.

\section{Appendix A: Transformation Boundaries}

Figure A1 shows a representation of the "tangent" construction lines used to establish the stresses and strains at the "nucleation" and "completion" of the $\mathrm{A} \rightarrow \mathrm{M}$ and $\mathrm{M} \rightarrow \mathrm{A}$ transformations. 


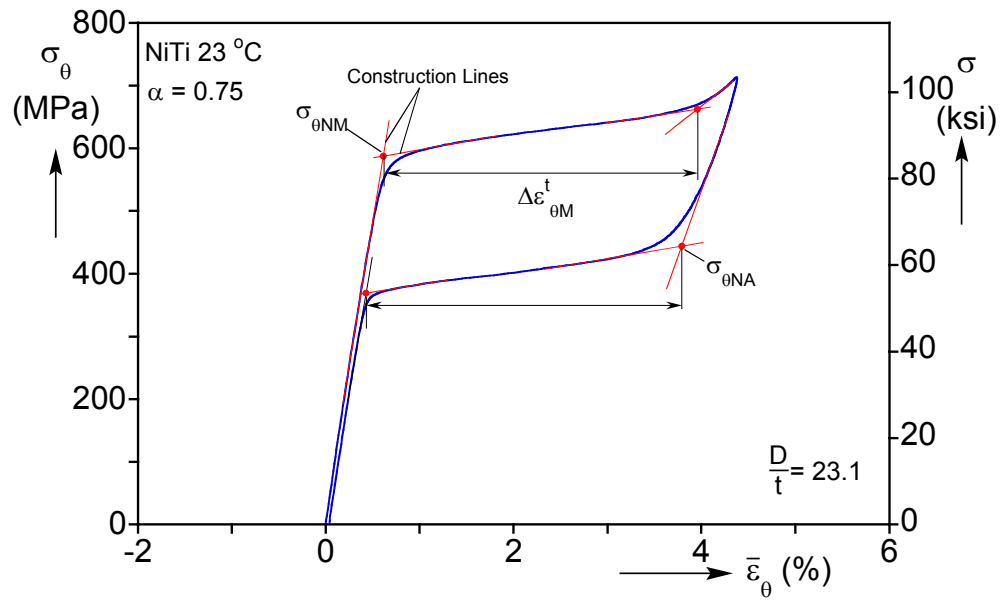

Fig. A1 Tangent construction lines for determining the onset and completion of transformations

\section{Appendix B: Tension Compression Asymmetry and Anisotropic "Yielding"}

To address tension-compression asymmetry in yielding observed for some metal alloys, Cazacu et al. [2006] (CPB06, see also Plunkett et al. [2008]) proposed the following isotropic yield function:

$$
\left(\left|s_{1}\right|-h s_{1}\right)^{k}+\left(\left|s_{2}\right|-h s_{2}\right)^{k}+\left(\left|s_{3}\right|-h s_{3}\right)^{k}=\text { cont. }
$$

where $s_{i}$ are the principal deviators of the Cauchy stress $\sigma$, the exponent $k$ is a positive integer, and $h$ is a constant determined from the ratio of yield stresses in uniaxial tension and compression. Anisotropy is introduced through the following linear transformation

$$
\Sigma=C s=C T \sigma
$$

producing the following orthotropic version of $(\mathrm{C} 1)$ :

$$
\Phi=\left(\left|\Sigma_{1}\right|-h \Sigma_{1}\right)^{k}+\left(\left|\Sigma_{2}\right|-h \Sigma_{2}\right)^{k}+\left(\left|\Sigma_{3}\right|-h \Sigma_{3}\right)^{k}
$$

where $\Sigma_{i}$ are the principal values of $\Sigma$. For a plane stress state in a reference Cartesian frame $(x, y, z)$, the relevant values of $\Sigma_{i j}$ are related to the in-plane stresses as follows: 


$$
\left\{\begin{array}{c}
\Sigma_{x x} \\
\Sigma_{y y} \\
\Sigma_{z z}
\end{array}\right\}=\frac{1}{3}\left[\begin{array}{lll}
C_{11} & C_{12} & C_{13} \\
C_{12} & C_{22} & C_{23} \\
C_{13} & C_{23} & C_{33}
\end{array}\right]\left[\begin{array}{ccc}
2 & -1 & -1 \\
-1 & 2 & -1 \\
-1 & -1 & 2
\end{array}\right]\left\{\begin{array}{c}
\sigma_{x x} \\
\sigma_{y y} \\
0
\end{array}\right\}
$$

and $\Sigma_{x y}=C_{66} \sigma_{x y}$.

$\Sigma_{i j}$ in (B3) are then used to establish the principal values $\Sigma_{i}$.

The martensite nucleation stresses from the biaxial experiments in Table 1 were used to develop a fit for the yield function (B2). For the purposes of this exercise the transformation stresses were established using an inelastic work criterion of $\Delta W^{t}=0.7 \mathrm{MPa}\left(\Delta W^{t}\right.$ is a measure of the nonlinearity in the initial response of A, similar to plastic work). The transformation stress under pure axial compression of 705.4 MPA is also included (from Jiang et al. 2015). The RHS of (B2) is assigned a constant value corresponding to the transformation stress under pure tension in the x-direction (see Eq. (12) in CPB06). The fitting was performed using the minimize algorithm included in the SciPy.optimize library based on the conjugate gradient method. The measured transformation stresses and the fit using the parameters listed in Table B1 are compared in Fig. 23.

Table B1 Anisotropy and asymmetry parameters for NiTi in the $\sigma_{X}-\sigma_{\theta}$ space

\begin{tabular}{|c|c|c|c|c|c|c|c|c|}
\hline$C_{11}$ & $C_{12}$ & $C_{13}$ & $C_{22}$ & $C_{23}$ & $C_{33}$ & $C_{66}$ & $h$ & $k$ \\
\hline 0.9615 & 0.1079 & 0.1489 & 0.9063 & 0.0378 & 1.138 & 1.0 & -0.2394 & 6 \\
\hline
\end{tabular}

It it worth noting that because of the limited data available, similar fits to the one shown in Fig. 23 were obtained using different exponents, $k$, resulting in different constants. Adoption of alternate fits had only small effects on the calculated band inclinations in Fig. 24. Clearly, a more unique fit of the transformation surface, although desirable, will require data in the rest of the stress space. 


\section{APPENDIX C: Austenitic Phase Elastic Constants}

The initial modulus of each axial and hoop stress-strain response measured is plotted against the stress ratio angle $\tan ^{-1} \alpha$ in Fig. $\mathrm{C} 1$. Included with dashed lines are the calculated stiffnesses using the material constants measured in the two uniaxial stress experiments, $E_{A}=66.3 \mathrm{GPa}$ and Poisson's ratio of $v_{A}=0.425$. The predictions follow the measured values very well for all values of $\alpha$, thus confirming the validity of assumed isotropic elastic behavior. Both $E_{A}$ and $v_{A}$ compare well to the values reported in Bechle and Kyriakides [2014].

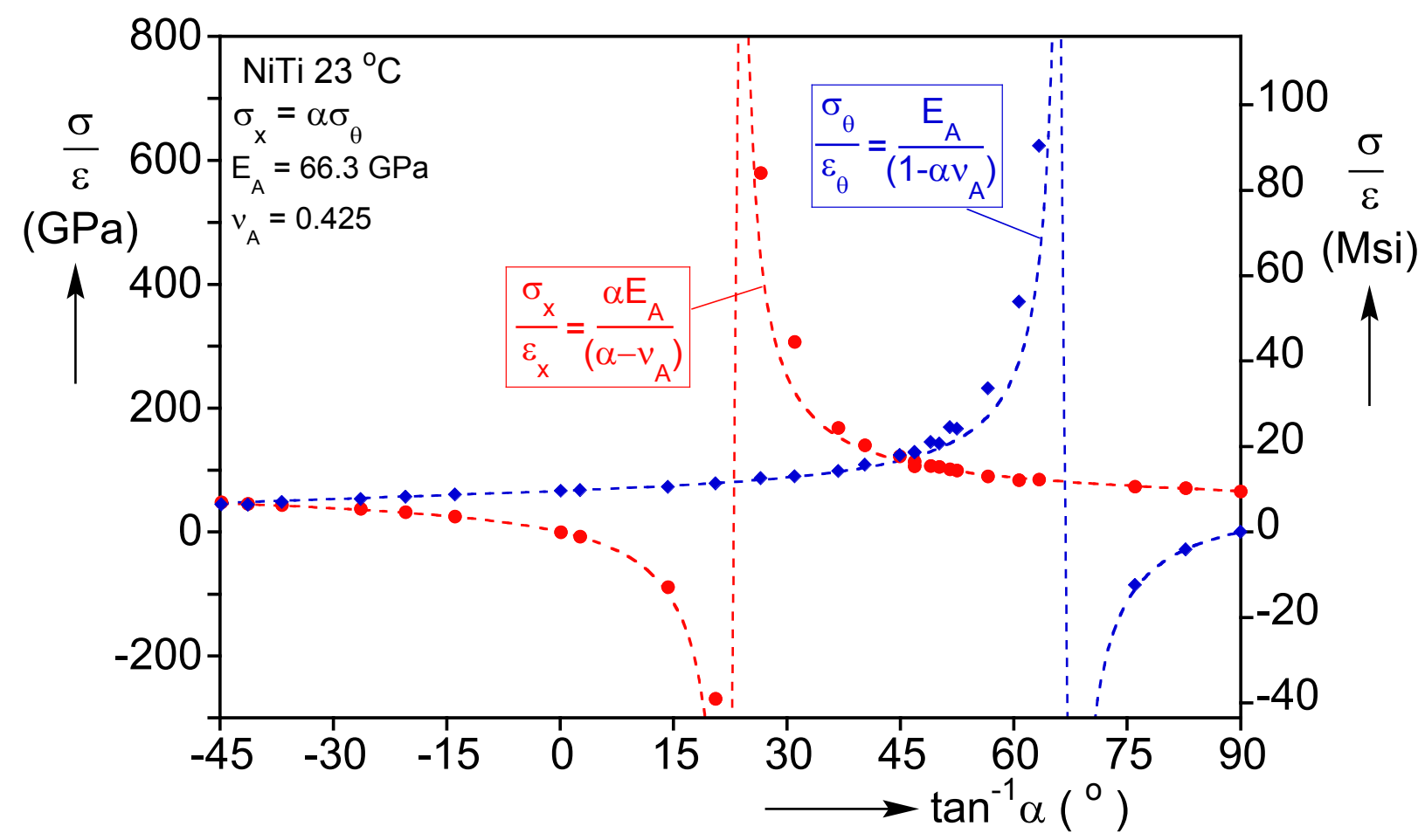

Fig. C1 The measured initial stiffness of each axial and hoop stress-strain response plotted against the stress ratio angle $\tan ^{-1} \alpha$ together with isotropic linear elasticity predictions. 


\section{Acknowledgments}

The work reported was conducted with the financial support of the National Science Foundation under grant no. CMMI-12000465. The authors acknowledge this support with thanks. NB acknowledges also to partial support from the University of Texas at Austin through a Thrust2000 graduate fellowship from the Cockrell School of Engineering.

\section{References}

Abeyaratne, R., Knowles, J.K. (2006). Evolution of phase transformations: A continuum theory. Cambridge University Press, New York.

Arghavani, J., Auricchio, F., Naghdabadi, R., Reali, A. and Sohrabpour, S. (2010). A 3-D phenomenological constitutive model for shape memory alloys under multiaxial loadings. Int'l $J$. Plasticity 26, 976-991.

Auricchio, F. and Petrini, L. (2004). A three-dimensional model describing stress-temperature induced solid phase transformations: solution algorithm and boundary value problems. Int'l J. Num. Meth. Eng. 61, 807-836.

Bechle, N.J. (2015). Inhomogeneous Deformations in NiTi Materials and Structures. PhD Dissertation, Engineering Mechanics, The University of Texas at Austin (in preparation).

Bechle, N.J., Kyriakides, S. (2014). Localization in NiTi tubes under bending. Int'l J. Solids Struct. 51, 967-980.

Bijlaard, P.P. (1940). Theory of local plastic deformations. Publication of the International Association of Bridge and Structural Engineers 6, 27-44.

Bouvet, C., Calloch, S., Lexcellent, C. (2002). Mechanical behavior of a Cu-Al-Be shape memory alloy under multiaxial proportional and nonproportional loadings. J. Eng. Mater. Technol. 124, 112-124.

Bouvet, C., Calloch, S., Taillard, K., Lexcellent, C. (2004). Experimental determination of initial surface of phase transformation of SMA. J. Physique IV - Proc. 115, 29-36.

Butler, J.F. (1962). Lüders front propagation in low-carbon steels. J. Mech. Physics of Solid 10, 313-334.

Cazacu, O., Plunkett, B., Barlat, F. (2006). Orthotropic yield criterion for hexagonal closed packed metals. Int. J. Plast. 22, 1171-1194.

Daly, S., Ravichandran, G., Bhattacharya, K. (2007). Stress-induced martensitic phase transformation in thin sheets of Nitinol. Acta Mat. 55, 3593-3600. 
Favier, D., Louche, H., Schlosser, P., Orgéas, L., Vacher, P., Debove, L. (2007). Homogeneous and heterogeneous mechanisms in an austenitic polycrystalline Ti-50.8 at.\% Ni thin tube under tension. Investigation via temperature and strain fields measurements. Acta Mat. 55, 5310-5322.

Feng, P., Sun, Q.P. (2006). Experimental investigation on macroscopic domain formation and evolution in polycrystalline NiTi microtubing under mechanical force. J. Mech. Physics Solid 54, 1568-1603.

Gall, K., Sehitoglu, H., Chumlyakov, Y.I. \& Kireeva, I.V. (1999). Tension-compression asymmetry of the stress-strain response in aged single crystals and polycrystalline NiTi. Acta Mater. 47, 1203-1217.

Gall, K., Sehitoglu, H. (1999). The role of texture in tension-compression asymmetry in polycrystalline NiTi. Int'l J. Plasticity 15, 69-92.

Govindjee, S., Hall, G.J. (2000). A computational model for shape memory alloys. Int'l. J. Solids Struct. 37, 735-760.

Govindjee, S., Miehe, C. (2001). A multi-variant martensitic phase transformation model: formulation and numerical implementation. Comp. Meth. Appl. Mech. Eng. 191, 215-238.

Grabe, C., Bruhns, O.T. (2009). Path dependence and multiaxial behavior of a polycrystalline NiTi alloy within the pseudoelastic and pseudoplastic temperature regimes. Int'l J. Plasticity $\mathbf{2 5}$, $513-545$.

Grolleau, V., Louche, H., Delobelle, V., Penin, A., Rio, G., Liu, Y. and Favier, D. (2011). Assessment of tension-compression asymmetry of NiTi using circular bulge testing of thin plates. Scripta Mat. 65, 347-350.

Hallai, J.F., Kyriakides, S. (2013). Underlying material response for Lüders-like instabilities. Int'l J. Plasticity 47, 1-12.

Hartl, D.J. and Lagoudas, D.C. (2009). Constitutive modeling and structural analysis considering simultaneous phase transformation and plastic yield in shape memory alloys. Smart Mater. Struct. 18, 104017.

Helm, D., Haupt, P., (2001). Thermomechanical behavior of shape memory alloys. Proc. SPIE Int'l Symposium on Smart Struct. and Materials 8, 302-313.

Hill, R. (1952). On discontinuous plastic states, with special reference to localized necking in thin sheets. J. Mech. Phys. Solids 1, 19-30.

Iadicola, M.A., Shaw, J.A. (2002). An Experimental setup for measuring unstable thermomechanical behavior of shape memory alloy wire. J. Intel. Mat. Syst. Struct. 13, 157-166.

Iadicola, M.A., Shaw, J.A. (2004). Rate and thermal sensitivities of unstable transformation 
behavior in a shape memory alloy. Int'l J. Plasticity 20, 577-605.

Jacobus, K., Sehitoglu, H. and Balzer, M. (1996). Effect of stress state on the stress-induced martensitic transformation of polycrystalline Ni-Ti Alloy. Metallu. Mater. Trans. A 27, 30663073.

Jiang, D., Bechle, N., Landis, C.M., Kyriakides, S. (2016). Buckling and recovery of NiTi tubes under axial compression. Int'l J. Solids \& Struct. 80, 52-63.

Kyriakides, S., Miller, J.E. (2000). On the propagation of Lüders bands in steel strips. ASME J. Appl. Mech. 67, 645-654.

Lexcellent, C., Vivet, A., Bouvet, C., Calloch, S., Blanc, P. (2002). Experimental and numerical determinations of the initial surface of phase transformation under biaxial loading in some polycrystalline shape-memory alloys. J. Mech. Physics Solids 50, 2717-2735.

Lexcellent, C., Blanc, P. (2004). Phase transformation yield surface determination for some shape memory alloys. Acta Mat. 52, 2317-2324.

Li, Z.Q., Sun Q.P. (2002). The initiation and growth of macroscopic martensite band in nanograined NiTi microtube under tension. Int'l J. Plasticity 18, 1481-1498.

Lim, T.J., McDowell, D.L. (1999). Mechanical behavior of a Ni-Ti shape memory alloy under axial-torsional proportional and nonproportional loading. J. Eng. Mater. Tech. 121, 9-18.

Liu, Y., Hoover, I., Xiang, H., Bataillard, L., and Miyazaki, S. (1999). Strain dependence of pseudoelastic hysteresis of NiTi. Metal. Mat. Trans. 30A, 1275-1282.

Mao, S., Luo, J., Zhang, Z., Wu, M., Liu, Y., Han, X. (2010). EBSD studies of the stress-induced B2-B19' martensitic transformation in NiTi tubes under uniaxial tension and compression. Acta Mat. 58, 3357-3366.

McNaney, J.M., Imbeni, V., Jung, Y., Papadopoulos, P., Ritchie, R.O. (2003). An experimental study of the superelastic effect in a shape-memory Nitinol alloy under biaxial loading. Mech. Materials 35, 969-986.

Michailidis, P.A., Triantafyllidis, N., Shaw, J.A., Grummon, D.S. (2009). Superelasticity and stability of a shape memory alloy hexagonal honeycomb under in-plane compression. Int'l J. Solids \& Structures 46, 2724-2738.

Nemat-Nasser, S., Choi, J.Y, Isaacs, J.B., Lischer, D.W. (2006). Quasi-static and dynamic buckling of thin cylindrical shape-memory shells. ASME J. Applied Mech. 73, 825-833.

Orgéas, L. and Favier, D. (1998). Stress-induced martensitic transfomation of a NiTi alloy in isothermal shear, tension and compression. Acta Mat. 46, 5579-5591. 
Patoor, E., El Armani, M., Eberhardt, A., Berveiller, M. (1995). Determination of the origin for the dissymmetry observed between tensile and compression tests on shape memory alloys. $J$. Physique IV 05, C2-495-C2-500.

Plunkett, B., Cazacu, O., Barlat, F. (2008). Orthotropic yield criteria for description of anisotropy in tension and compression in sheet metalshexagonal closed packed metals. Int. J. Plast. 24, 847866.

Popov, P., Lagoudas, D.C. (2007). A 3-D constitutive model for shape memory alloys incorporating pseudoelasticity and detwinning of self-accommodated martensite. Int'l $J$. Plasticity 23, 1679-1720.

Rahman, M.A, Qiu, J., Tani, J. (2001). Buckling and postbuckling characteristics of the superelastic SMA columns. Int'l J. Solids \& Structures 38, 9253-9265.

Reedlunn, B., Churchill, C.B., Nelson, E.E., Shaw, J.A., Daly, S.H. (2014). Tension, compression and bending of superelastic shape memory tubes. J. Mech. Physics Solids 63, 506537.

Rogueda, C., Lexcellent, C., Bocher, L. (1996). Experimental study of pseudoelastic behaviour of a $\mathrm{Cu} \mathrm{Zn}$ AI polycrystalline shape memory alloy under tension-torsion proportional and nonproportional loading tests. Archives Mech. 48, 1025 - 1047.

Sehitoglu, H., Karaman, I., Anderson, R., Zhang, X., Gall, K., Maier, H.J. and Chumlyakov, Y. (2000). Compressive response of NiTi single crystals. Acta Mat. 48, 3311-3326.

Shaw, J.A. and Kyriakides, S. (1995). Thermomechanical aspects of NiTi. J. Mechanics Physics Solids 43, 1243-1281.

Shaw, J.A. and Kyriakides, S. (1997). On the nucleation and propagation of phase transformation fronts in a NiTi alloy. Acta Mat. 45, 683-700.

Shaw, J.A. and Kyriakides, S. (1998). Initiation and propagation of localized deformation in elasto-plastic strips under uniaxial tension. Int'l J. Plast. 13, 837-871.

Shaw, J.A., Reedlunn, B., Daly, S. (2013). Instabilities during tension-torsion of superelastic NiTi tubes. Oral presentation at SES 50th annual Technical Meeting and AMD-ASME Summer Meeting. Brown University, Providence, RI, July 28-Aug. 1, 2013.

Sun, Q.P., Li, Z.Q. (2002). Phase transformation in superelastic NiTi polycrystalline micro-tubes under tension and torsion-from localization to homogeneous deformation. Int'l J. Solids \& Structures 39, 3797-3809.

Tang, Z., Li, D. (2012). Quasi-static axial buckling behavior of NiTi thin-walled cylindrical shells. Thin Walled Structures 51, 130-138. 
Thamburaja, P., Anand, L. (2002). Superelastic behavior in tension-torsion of an initiallytextured Ti-Ni shape-memory alloy. Int'l J. Plasticity 18, 1607-1617.

Wasilewski, R.J. (1971). The effects of applied stress on the martensitic transformation in NiTi. Metallu. Trans. 2, 2973-2981.

\section{Figure Legends}

Fig. 1 Internal pressure-axial load biaxial test facility. (a) Schematic and (b) photograph of the grips and specimen.

Fig. 2 Detail of gripped end of the $6.35 \mathrm{~mm}$ NiTi tubular specimen.

Fig. 3 Stress-average strains recorded in an isothermal uniaxial axial tension test on a NiTi tube at $23{ }^{\circ} \mathrm{C}$.

Fig. 4 Sequences of axial and hoop strain contours from DIC corresponding to the numbered bullets marked on the tensile responses in Fig. 3.

Fig. 5 Histograms of axial strain that show the degree of strain inhomogeneity in the FOV for the 20 strain images in Fig. 4.

Fig. 6 Position $(x)$ of transformation fronts vs. time $(t)$ during the unloading of the uniaxial tension experiment in Fig. 3.

Fig. 7 Expanded view of an inclined helical martensitic transformation front.

Fig. 8 Prescribed radial stress paths from representative experiments on NiTi tubes using setup in Fig. 1 and 2.

Fig. 9 Stress-average strains recorded in biaxial test with $\sigma_{x}=0.375 \sigma_{\theta}$.

Fig. 10 Sequences of axial and hoop strain contours from DIC corresponding to the numbered bullets marked on the responses in Fig. 9.

Fig. 11 Histograms of hoop strain that show the degree of strain inhomogeneity in the FOV for the 20 strain images in Fig. 10.

Fig. 12 Results from a uniaxial hoop tension test. (a) Stress-average strain responses and (b) sequences of axial and hoop strain contours from DIC corresponding to the numbered bullets marked on the responses in (a).

Fig. 13 Results from a biaxial test with $\sigma_{X}=-0.374 \sigma_{\theta}$. (a) Stress-average strain responses and (b) sequences of axial and hoop strain contours from DIC corresponding to the numbered bullets marked on the responses in (a). 
Fig. 14 Results from biaxial test with $\sigma_{X}=-0.75 \sigma_{\theta}$. (a) Stress-average strain responses and (b) sequences of axial and hoop strain contours from DIC corresponding to the numbered bullets marked on the responses in (a).

Fig. 15 Results from a biaxial test with $\sigma_{X}=0.60 \sigma_{\theta}$. (a) Stress-average strain responses and (b) sequences of axial and hoop strain contours from DIC corresponding to the numbered bullets marked on the responses in (a).

Fig. 16 Results from a biaxial test with $\sigma_{X}=0.85 \sigma_{\theta}$. (a) Stress-average strain responses and (b) sequences of axial and hoop strain contours from DIC corresponding to the numbered bullets marked on the responses in (a).

Fig. 17 Results from a biaxial test with $\sigma_{X}=1.0 \sigma_{\theta}$. (a) Stress-average strain responses and (b) sequences of axial and hoop strain contours from DIC corresponding to the numbered bullets marked on the responses in (a).

Fig. 18 Results from a biaxial test with $\sigma_{X}=1.07 \sigma_{\theta}$. (a) Stress-average strain responses and (b) sequences of axial and hoop strain contours from DIC corresponding to the numbered bullets marked on the responses in (a).

Fig. 19 Results from a biaxial test with $\sigma_{X}=1.20 \sigma_{\theta}$. (a) Stress-average strain responses and (b) sequences of axial and hoop strain contours from DIC corresponding to the numbered bullets marked on the responses in (a).

Fig. 20 Results from a biaxial test with $\sigma_{x}=1.30 \sigma_{\theta}$. (a) Stress-average strain responses and (b) sequences of axial and hoop strain contours from DIC corresponding to the numbered bullets marked on the responses in (a).

Fig. 21 Results from a biaxial test with $\sigma_{X}=4.0 \sigma_{\theta}$. (a) Stress-average strain responses and (b) sequences of axial and hoop strain contours from DIC corresponding to the numbered bullets marked on the responses in (a).

Fig. 22 Localization band in a plastic material under plane stress.

Fig. 23 Locus of martensite transformation stresses from the biaxial experiments performed and a fit based on CPB06 (Cazacu et al., 2006).

Fig. 24 Measured inclination of martensitic localization band to axial direction vs. stress ratio angle. Included are predictions based on Eq. (5) for the CPB06 and von Mises transformation surfaces.

Fig. 25 Representative stress-average strain responses for biaxial stress states with $\alpha \leq 1.0$. (a) $\sigma_{X}-\bar{\varepsilon}_{X}$ and (b) $\sigma_{\theta}-\bar{\varepsilon}_{\theta}$. 
Fig. 26 Representative stress-average strain responses for biaxial stress states with $\alpha \geq 1$.0. (a) $\sigma_{X}-\bar{\varepsilon}_{X}$ and (b) $\sigma_{\theta}-\bar{\varepsilon}_{\theta}$.

Fig. 27 Loci of measured stresses at the nucleation and "completion" of M and A transformations and fitted surfaces.

Fig. 28 Measured axial vs. hoop strains at the nucleation and "completion" of (a) M and (b) A transformations.

Fig. 29 Measured transformation dissipation energy vs. stress ratio angle. 